\title{
Erratum to: $L^{2}$-norm transformation for improving $k$-means clustering
}

Finding a suitable model by range transformation for novel data analysis

Piyush Kumar Sharma ${ }^{1}$ - Gary Holness ${ }^{2}$

Published online: 21 August 2017

(C) Springer International Publishing AG 2017

\section{Erratum to: Int J Data Sci Anal (2017) 3:247-266 \\ DOI 10.1007/s41060-017-0054-1}

The content of Table 1 was inadvertently published twice, once in Table 1 and again in Table 2. Please find below Tables 1 and 2 with the correct content.

The online version of the original article can be found under doi:10.1007/s41060-017-0054-1.

Piyush Kumar Sharma

pksharma12@students.desu.edu

Gary Holness

gholness@desu.edu

1 Department of Mathematical Sciences, Delaware State University, Dover, DE 19904, USA

2 Department of Computer and Information Sciences, Delaware State University, Dover, DE 19904, USA 
Table 1 CJSD kernels SVM classification accuracies

\begin{tabular}{|c|c|c|c|c|}
\hline Datasets & Kernel & A.M. $(\%)$ & G.M. (\%) & H.M. (\%) \\
\hline \multirow{2}{*}{ DOOO vs. D010 } & Amplify & $59.6 \pm 0.9$ & $59.5 \pm 0.4$ & $60.2 \pm 1.1$ \\
\hline & Scaled & $57.8 \pm 0.9$ & $82.7 \pm 0.4$ & $57 \pm 0.7$ \\
\hline סבחמ צy & Amplify & $68.3 \pm 0.7$ & $68 \pm 1.4$ & $67.3 \pm 1.0$ \\
\hline Duou vs. DuZu & Scaled & $68.6 \pm 0.9$ & $83.2 \pm 0.8$ & $83.3 \pm 0.8$ \\
\hline & Amplify & $78.1 \pm 1.0$ & $81.6 \pm 0.9$ & $81.9 \pm 0.4$ \\
\hline D000 vs. D030 & Scaled & $81.6 \pm 0.9$ & $83.7 \pm 0.7$ & $83.8 \pm 0.9$ \\
\hline D000 ys, D040 & Amplify & $88.7 \pm 0.9$ & $87.5 \pm 0.8$ & $87.8 \pm 1.0$ \\
\hline $1000 \mathrm{Vs} .10040$ & Scaled & $88.8 \pm 0.7$ & $83.1 \pm 0.8$ & $83.5 \pm 0.6$ \\
\hline & Amplify & $92.5 \pm 0.5$ & $92.3 \pm 0.5$ & $92.4 \pm 0.7$ \\
\hline DU00 vs. Duso & Scaled & $91.8 \pm 0.9$ & $86.9 \pm 1.3$ & $83.7 \pm 0.7$ \\
\hline & Amplify & $95.7 \pm 0.4$ & $95.8 \pm 0.6$ & $95.7 \pm 0.4$ \\
\hline DU00 vs. DU60 & Scaled & $95.7 \pm 0.4$ & $93.6 \pm 1.8$ & $86.6 \pm 1.9$ \\
\hline & Amplify & $97.3 \pm 0.2$ & $97.4 \pm 0.4$ & $97.5 \pm 0.3$ \\
\hline Dour vs. DO & Scaled & $95.7 \pm 0.2$ & $97.4 \pm 0.4$ & $96.5 \pm 1.1$ \\
\hline & Amplify & $98 \pm 0.3$ & $98 \pm 0.3$ & $98 \pm 0.3$ \\
\hline Duou vs. Dusu & Scaled & $98 \pm 0.3$ & $98.1 \pm 0.3$ & $98.2 \pm 0.3$ \\
\hline & Amplify & $98.9 \pm 0.2$ & $98.9 \pm 0.2$ & $98.9 \pm 0.3$ \\
\hline & Scaled & $98.9 \pm 0.2$ & $98.9 \pm 0.2$ & $99.1 \pm 0.3$ \\
\hline & Amplify & $63.2 \pm 1.3$ & $95.6 \pm 0.8$ & $63.8 \pm 1.3$ \\
\hline DU10 vs. DU20 & Scaled & $63.2 \pm 1.3$ & $69.3 \pm 1.1$ & $68.7 \pm 1.0$ \\
\hline D010 vs D030 & Amplify & $72.5 \pm 1.3$ & $77.1 \pm 0.9$ & $78.1 \pm 0.8$ \\
\hline Doto vs. Doso & Scaled & $74.7 \pm 1.1$ & $79.1 \pm 0.8$ & $75.7 \pm 0.7$ \\
\hline & Amplify & $82.8 \pm 1.2$ & $84.7 \pm 0.9$ & $85.6 \pm 0.7$ \\
\hline Doto vs. D040 & Scaled & $82.5 \pm 0.9$ & $80 \pm 0.9$ & $74.4 \pm 1.2$ \\
\hline D010 ys D050 & Amplify & $89.2 \pm 0.6$ & $89.5 \pm 0.7$ & $90.6 \pm 0.5$ \\
\hline DUto vs. Duso & Scaled & $87.8 \pm 0.7$ & $81 \pm 1$ & $75.5 \pm 0.7$ \\
\hline D010 ys. D060 & Amplify & $94.5 \pm 0.3$ & $93.6 \pm 0.7$ & $93.3 \pm 0.3$ \\
\hline Dotiog. & Scaled & $93.3 \pm 0.4$ & $80.4 \pm 0.9$ & $73.1 \pm 1.2$ \\
\hline & Amplify & $96.6 \pm 0.4$ & $96.2 \pm 0.4$ & $96.2 \pm 0.4$ \\
\hline DO10 vs. DO & Scaled & $95.7 \pm 0.6$ & $80.6 \pm 0.9$ & $74.2 \pm 1.1$ \\
\hline D010 vs. Do80 & Amplify & $97.5 \pm 0.4$ & $96.9 \pm 0.2$ & $96.6 \pm 0.4$ \\
\hline Doto Vs. Dod & Scaled & $96.6 \pm 0.4$ & $81.3 \pm 0.5$ & $72.9 \pm 0.6$ \\
\hline חס9010 & Amplify & $98.7 \pm 0.2$ & $98.1 \pm 0.3$ & $98 \pm 0.23$ \\
\hline Doto vs. Do90 & Scaled & $97.9 \pm 0.3$ & $81.7 \pm 0.9$ & $75.7 \pm 0.7$ \\
\hline & Amplify & $61.6 \pm 0.6$ & $63.8 \pm 1.0$ & $63.3 \pm 1.2$ \\
\hline Duzu vs. Dusu & Scaled & $61.4 \pm 1.1$ & $64.7 \pm 0.8$ & $64.1 \pm 1.4$ \\
\hline D020 ys, D040 & Amplify & $70 \pm 1.0$ & $74.3 \pm 0.6$ & $74.5 \pm 0.6$ \\
\hline $020 \mathrm{vs} .10040$ & Scaled & $72.8 \pm 0.6$ & $74.2 \pm 0.6$ & $68.2 \pm 0.8$ \\
\hline D020 ys D050 & Amplify & $76.8 \pm 1.0$ & $80.9 \pm 0.6$ & $80.5 \pm 0.9$ \\
\hline DO20 vs. DUso & Scaled & $80 \pm 0.7$ & $80.5 \pm 0.8$ & $75.7 \pm 0.7$ \\
\hline & Amplify & $86.9 \pm 0.7$ & $88.8 \pm 0.9$ & $88.2 \pm 0.9$ \\
\hline D020 vs. D060 & Scaled & $87.5 \pm 0.8$ & $87.4 \pm 1.2$ & $76.8 \pm 1.7$ \\
\hline & Amplify & $91.4 \pm 0.6$ & $91.6 \pm 0.6$ & $91.6 \pm 0.7$ \\
\hline $020 \mathrm{Vs} .10070$ & Scaled & $91.3 \pm 0.7$ & $90.7 \pm 0.7$ & $81.1 \pm 2.2$ \\
\hline D020 ys Do80 & Amplify & $94.5 \pm 0.6$ & $93.9 \pm 0.5$ & $94.2 \pm 0.6$ \\
\hline Duzu vs. Dusu & Scaled & $93.7 \pm 0.6$ & $90.9 \pm 0.8$ & $83.7 \pm 1.3$ \\
\hline D020 ys D090 & Amplify & $96.5 \pm 0.5$ & $95.8 \pm 0.5$ & $95.3 \pm 0.4$ \\
\hline 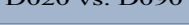 & Scaled & $95.7 \pm 0.5$ & $94.6 \pm 0.5$ & $87.2 \pm 0.8$ \\
\hline & Amplify & $56.4 \pm 1.0$ & $59.2 \pm 1.0$ & $58.1 \pm 1.1$ \\
\hline D030 vs. D040 & Scaled & $57.8 \pm 1.4$ & $59.5 \pm 0.9$ & $57.7 \pm 0.6$ \\
\hline D030 ys D050 & Amplify & $61.8 \pm 1.1$ & $67.1 \pm 0.9$ & $66.2 \pm 0.8$ \\
\hline D030 vs. DOso & Scaled & $67 \pm 1.2$ & $67.5 \pm 0.7$ & $67.5 \pm 0.8$ \\
\hline D030 ys D060 & Amplify & $74.1 \pm 0.8$ & $77.4 \pm 0.9$ & $76.8 \pm 0.6$ \\
\hline D030 vs. D060 & Scaled & $77.2 \pm 0.8$ & $77.2 \pm 0.9$ & $75.5 \pm 0.9$ \\
\hline D030 ys & Amplify & $81.3 \pm 1.0$ & $82.9 \pm 0.7$ & $83.1 \pm 0.5$ \\
\hline D030 vs. D0/0 & Scaled & $84 \pm 0.7$ & $82.7 \pm 0.7$ & $80.7 \pm 0.6$ \\
\hline 0080 & Amplify & $86.4 \pm 0.7$ & $88.3 \pm 0.6$ & $87 \pm 1.0$ \\
\hline D080 & Scaled & $88.6 \pm 0.7$ & $87.8 \pm 0.6$ & $82.9 \pm 1.2$ \\
\hline D030 ys Do90 & Amplify & $90.1 \pm 0.6$ & $91.4 \pm 0.8$ & $90.6 \pm 0.6$ \\
\hline D030 vs. D090 & Scaled & $91.3 \pm 0.4$ & $89.5 \pm 0.7$ & $86.4 \pm 0.9$ \\
\hline D040 ys D050 & Amplify & $54.2 \pm 1.0$ & $56.4 \pm 1.0$ & $56.4 \pm 0.7$ \\
\hline Dutov. Doso & Scaled & $56.4 \pm 1.1$ & $56.6 \pm 1.1$ & $56.8 \pm 0.9$ \\
\hline & Amplify & $62.4 \pm 0.7$ & $66.9 \pm 1.0$ & $67 \pm 0.9$ \\
\hline & Scaled & $66.8 \pm 0.8$ & $66.2 \pm 1.0$ & $66.2 \pm 1.0$ \\
\hline & Amplify & $69.8 \pm 0.9$ & $74.5 \pm 1.0$ & $74.2 \pm 0.9$ \\
\hline D040 vs. D070 & Scaled & $75.1 \pm 0.6$ & $74.1 \pm 0.9$ & $72.5 \pm 1.0$ \\
\hline 040 ys, D080 & Amplify & $76.4 \pm 0.9$ & $80.5 \pm 1.0$ & $80.5 \pm 0.8$ \\
\hline & Scaled & $81.6 \pm 0.8$ & $80.1 \pm 1.1$ & $77.3 \pm 0.7$ \\
\hline ys $\mathrm{J}$ & Amplify & $81.2 \pm 0.8$ & $84.6 \pm 0.4$ & $84.4 \pm 0.8$ \\
\hline 40 vs. D & Scaled & $85.1 \pm 0.9$ & $84 \pm 0.5$ & $81.1 \pm 0.7$ \\
\hline D050 ys D060 & Amplify & $54.6 \pm 0.5$ & $56.2 \pm 0.7$ & $57 \pm 0.9$ \\
\hline D050 vs. D060 & Scaled & $55.1 \pm 1$ & $57 \pm 0.7$ & $57.6 \pm 0.7$ \\
\hline & Amplify & $60.6 \pm 1.1$ & $66 \pm 0.9$ & $64.2 \pm 0.6$ \\
\hline & Scaled & $66.2 \pm 1.1$ & $65.9 \pm 1.0$ & $64.4 \pm 0.7$ \\
\hline$D 050$ ys & Amplify & $67 \pm 0.7$ & $72.8 \pm 1.2$ & $71.4 \pm 0.8$ \\
\hline $030 \mathrm{Vs} . \mathrm{D} 080$ & Scaled & $74.9 \pm 0.7$ & $73.2 \pm 1.2$ & $70.1 \pm 0.9$ \\
\hline & Amplify & $72.9 \pm 0.8$ & $78.3 \pm 0.8$ & $77.8 \pm 0.6$ \\
\hline (5) & Scaled & $79 \pm 0.8$ & $78.3 \pm 0.9$ & $75.1 \pm 0.7$ \\
\hline & Amplify & $53.6 \pm 0.8$ & $56.4 \pm 0.9$ & $56.3 \pm 0.9$ \\
\hline D060 vs. D07 & Scaled & $57.9 \pm 1$ & $56.4 \pm 0.7$ & $56.4 \pm 0.7$ \\
\hline D060 ys D080 & Amplify & $59.7 \pm 0.8$ & $63.6 \pm 0.7$ & $62.1 \pm 0.8$ \\
\hline D060 vs. D080 & Scaled & $64.8 \pm 0.9$ & $63.8 \pm 0.6$ & $61.2 \pm 1.2$ \\
\hline & Amplify & $65.8 \pm 1.2$ & $69.9 \pm 0.9$ & $68.9 \pm 0.9$ \\
\hline & Scaled & $72 \pm 1.4$ & $69.8 \pm 0.9$ & $68 \pm 0.9$ \\
\hline & Amplify & $54.6 \pm 1.4$ & $56.3 \pm 0.6$ & $55.5 \pm 1.2$ \\
\hline D070 vs. D080 & Scaled & $56.5 \pm 1.1$ & $56.3 \pm 0.9$ & $55.2 \pm 1.1$ \\
\hline 70 ys & Amplify & $57.3 \pm 0.8$ & $62.2 \pm 0.8$ & $62.2 \pm 1.1$ \\
\hline /O vs. DO & Scaled & $61.9 \pm 1$ & $62.5 \pm 0.9$ & $61.9 \pm 1.1$ \\
\hline & Amplify & $53.1 \pm 1.0$ & $52.3 \pm 0.8$ & $52.9 \pm 0.8$ \\
\hline D080 vs. D090 & Scaled & $53.9 \pm 0.7$ & $50.6 \pm 0.6$ & $53.2 \pm 1.1$ \\
\hline
\end{tabular}

Table 2 RBF kernels SVM classification accuracies

\begin{tabular}{|c|c|c|c|c|}
\hline Datasets & Kernel & A.M. (\%) & G.M. $(\%)$ & H.M. (\%) \\
\hline \multirow{2}{*}{ Do00 vs. Do10 } & Amplify & $50.4 \pm 1.5$ & $51 \pm 1.1$ & $51.9 \pm 0.9$ \\
\hline & Scaled & $50.4 \pm 1.5$ & $51 \pm 1.1$ & $51.9 \pm 0.9$ \\
\hline & Amplify & $52 \pm 0.8$ & $50 \pm 0.8$ & $51.6 \pm 0.9$ \\
\hline Duto vs. $00 \angle 0$ & Scaled & $52 \pm 0.8$ & $50 \pm 0.8$ & $51.6 \pm 0.9$ \\
\hline & Amplify & $49.8 \pm 1.2$ & $51.5 \pm 1.6$ & $49.3 \pm 1.9$ \\
\hline D000 vs. D030 & Scaled & $49.8 \pm 1.2$ & $51.5 \pm 1.6$ & $49.3 \pm 1.9$ \\
\hline & Amplify & $53.5 \pm 1.8$ & $5.1 \pm 1.2$ & $52.5 \pm 0.9$ \\
\hline Du00 vs. D040 & Scaled & $53.5 \pm 1.8$ & $51 \pm 1.2$ & $52.5 \pm 0.9$ \\
\hline 2000 ys D050 & Amplify & $52 \pm 0.7$ & $51.8 \pm 1.3$ & $52.6 \pm 1.5$ \\
\hline Do00 Vs. Dosu & Scaled & $52 \pm 0.7$ & $51.8 \pm 1.3$ & $52.6 \pm 1.5$ \\
\hline D000 ys. D06 & Amplify & $52.5 \pm 1.4$ & $50.9 \pm 0.7$ & $52.2 \pm 1.2$ \\
\hline Duov vs. Doou & Scaled & $52.5 \pm 1.4$ & $50.9 \pm 0.7$ & $52.2 \pm 1.2$ \\
\hline & Amplify & $53.8 \pm 1.4$ & $53.9 \pm 1.1$ & $54.3 \pm 1.2$ \\
\hline D000 vs. Do/ & Scaled & $53.8 \pm 1.4$ & $53.9 \pm 1.1$ & $54.3 \pm 1.2$ \\
\hline & Amplify & $54.3 \pm 1.9$ & $55.2 \pm 2.1$ & $57.5 \pm 1.5$ \\
\hline D000 vs. Do80 & Scaled & $54.3 \pm 1.9$ & $55.2 \pm 2.1$ & $57.5 \pm 1.5$ \\
\hline DOO0 ys, D & Amplify & $55.6 \pm 1.2$ & $57 \pm 2.3$ & $56.4 \pm 1.8$ \\
\hline & Scaled & $55.6 \pm 1.2$ & $57 \pm 2.3$ & $56.4 \pm 1.8$ \\
\hline & Amplify & $50.3 \pm 1.3$ & $51 \pm 0.9$ & $50.6 \pm 1.1$ \\
\hline Do10 vs & Scaled & $50.3 \pm 1.3$ & $51 \pm 0.9$ & $50.6 \pm 1.1$ \\
\hline D010 ys D030 & Amplify & $49.8 \pm 1.1$ & $48.4 \pm 1.2$ & $51 \pm 1.6$ \\
\hline Doitus. Doso & Scaled & $49.8 \pm 1.1$ & $48.4 \pm 1.2$ & $51 \pm 1.6$ \\
\hline & Amplify & $52.3 \pm 0.9$ & $53 \pm 1.1$ & $51.5 \pm 0.7$ \\
\hline & Scaled & $52.3 \pm 0.9$ & $53 \pm 1.1$ & $51.5 \pm 0.7$ \\
\hline & Amplify & $51.9 \pm 1.5$ & $52.8 \pm 0.9$ & $54.2 \pm 1.0$ \\
\hline Duto vs. Duso & Scaled & $51.9 \pm 1.5$ & $52.8 \pm 0.9$ & $54.2 \pm 1.0$ \\
\hline D010 ys, D060 & Amplify & $53 \pm 1.0$ & $54.2 \pm 1.2$ & $53.2 \pm 1.2$ \\
\hline DUto vs. DU60 & Scaled & $53 \pm 1.0$ & $54.2 \pm 1.2$ & $53.2 \pm 1.2$ \\
\hline D010 vs D07 & Amplify & $53.5 \pm 1.5$ & $55.1 \pm 1.1$ & $54 \pm 0.8$ \\
\hline $0010 \mathrm{v}-2-2$ & Scaled & $53.5 \pm 1.5$ & $55.1 \pm 1.1$ & $54 \pm 0.8$ \\
\hline Do10 & Amplify & $54.2 \pm 1.4$ & $54.6 \pm 1.7$ & $53.7 \pm 1.2$ \\
\hline Doto Vs. Dodo & Scaled & $54.2 \pm 1.4$ & $54.6 \pm 1.7$ & $53.7 \pm 1.2$ \\
\hline & Amplify & $55.2 \pm 1.8$ & $56.1 \pm 1.5$ & $53.2 \pm 1.7$ \\
\hline DU10 vs. Dug0 & Scaled & $55.2 \pm 1.8$ & $56.1 \pm 1.5$ & $53.2 \pm 1.7$ \\
\hline 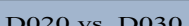 & Amplify & $52.4 \pm 0.8$ & $51.1 \pm 1.1$ & $51.4 \pm 1.0$ \\
\hline Dozo vas. Doso & Scaled & $52.4 \pm 0.8$ & $51.1 \pm 1.1$ & $51.4 \pm 1.0$ \\
\hline & Amplify & $50.9 \pm 1.0$ & $51.4 \pm 0.8$ & $51.5 \pm 0.9$ \\
\hline 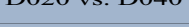 & Scaled & $50.9 \pm 1.0$ & $51.4 \pm 0.8$ & $51.5 \pm 0.9$ \\
\hline Do20 vs. & Amplify & $51.7 \pm 1.2$ & $51.6 \pm 0.5$ & $51.9 \pm 1.0$ \\
\hline DO20 vs. & Scaled & $51.7 \pm 1.2$ & $51.6 \pm 0.5$ & $51.9 \pm 1.0$ \\
\hline D020 ys D060 & Amplify & $53 \pm 1.3$ & $51.7 \pm 0.9$ & $50.6 \pm 0.4$ \\
\hline Do20 Vs. Do60 & Scaled & $53 \pm 1.3$ & $51.7 \pm 0.9$ & $50.6 \pm 0.4$ \\
\hline & Amplify & $52.8 \pm 1.1$ & $53.5 \pm 1.3$ & $54.1 \pm 1.2$ \\
\hline & Scaled & $52.8 \pm 1.0$ & $53.5 \pm 1.3$ & $54.1 \pm 1.2$ \\
\hline & Amplify & $53 \pm 1.2$ & $53 \pm 1.0$ & $55 \pm 1.0$ \\
\hline Duzo vs. Duso & Scaled & $53 \pm 1.2$ & $53 \pm 1.0$ & $55 \pm 1.0$ \\
\hline 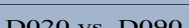 & Amplify & $56.3 \pm 1.5$ & $55 \pm 1.5$ & $56 \pm 1.5$ \\
\hline Du20 vs. Dugu & Scaled & $56.3 \pm 1.5$ & $55 \pm 1.5$ & $56 \pm 1.5$ \\
\hline & Amplify & $51.7 \pm 1.3$ & $50.5 \pm 0.7$ & $52.1 \pm 0.9$ \\
\hline Doso vs. D & Scaled & $51.7 \pm 1.3$ & $50.5 \pm 0.7$ & $52.1 \pm 0.9$ \\
\hline ys & Amplify & $50.7 \pm 0.8$ & $52 \pm 1.2$ & $49.2 \pm 1.4$ \\
\hline Do30 vs. Doso & Scaled & $50.7 \pm 0.8$ & $52 \pm 1.2$ & $49.2 \pm 1.4$ \\
\hline D030 vs & Amplify & $51.9 \pm 0.8$ & $50.4 \pm 0.4$ & $52.5 \pm 1.0$ \\
\hline & Scaled & $51.9 \pm 0.8$ & $50.4 \pm 0.4$ & $52.5 \pm 1.0$ \\
\hline & Amplify & $53.3 \pm 1.2$ & $52.3 \pm 0.8$ & $52 \pm 1.0$ \\
\hline Duso vs. DU/0 & Scaled & $53.3 \pm 1.2$ & $52.3 \pm 0.8$ & $52 \pm 1.0$ \\
\hline & Amplify & $54.5 \pm 1.4$ & $54.4 \pm 1.2$ & $54.4 \pm 1.7$ \\
\hline & Scaled & $54.5 \pm 1.4$ & $54.4 \pm 1.2$ & $54.4 \pm 1.7$ \\
\hline D036 $>2+2$ & Amplify & $55.1 \pm 1.5$ & $56.2 \pm 1.1$ & $54.4 \pm 1.5$ \\
\hline & Scaled & $55.5 \pm 1.5$ & $56.2 \pm 1.1$ & $54.4 \pm 1.5$ \\
\hline D040 ys D050 & Amplify & $50.3 \pm 1.6$ & $49.3 \pm 0.9$ & $50.1 \pm 1.0$ \\
\hline DU40 Vs. DOSO & Scaled & $50.3 \pm 0.6$ & $49.3 \pm 0.9$ & $50.1 \pm 1.0$ \\
\hline & Amplify & $50.5 \pm 0.3$ & $51.7 \pm 0.7$ & $52.3 \pm 0.8$ \\
\hline & Scaled & $50.5 \pm 0.3$ & $51.7 \pm 0.7$ & $52.3 \pm 0.8$ \\
\hline D040 ys $\mathrm{D}$ & Amplify & $51 \pm 1.1$ & $51.3 \pm 1.1$ & $52.5 \pm 1.4$ \\
\hline Du40 vs. Du / & Scaled & $51 \pm 1.1$ & $51.3 \pm 1.1$ & $52.5 \pm 1.4$ \\
\hline & Amplify & $52.7 \pm 0.8$ & $53.3 \pm 1.2$ & $52.2 \pm 1.2$ \\
\hline D040 vs. D080 & Scaled & $52.7 \pm 0.8$ & $53.3 \pm 1.2$ & $52.2 \pm 1.2$ \\
\hline & Amplify & $52.6 \pm 1.2$ & $52.9 \pm 1.3$ & $55.6 \pm 2.9$ \\
\hline & Scaled & $52.6 \pm 1.2$ & $52.9 \pm 1.3$ & $55.6 \pm 2.9$ \\
\hline ys Do6 & Amplify & $50.9 \pm 0.8$ & $52.3 \pm 1.0$ & $49.7 \pm 0.6$ \\
\hline D0so vs. D060 & Scaled & $50.9 \pm 0.8$ & $52.3 \pm 1.0$ & $49.7 \pm 0.6$ \\
\hline & Amplify & $50.1 \pm 1.2$ & $51.9 \pm 0.7$ & $50.1 \pm 0.5$ \\
\hline DOSO vs. Do & Scaled & $50.1 \pm 1.2$ & $51.9 \pm 0.7$ & $50.1 \pm 0.5$ \\
\hline 0050 ys D080 & Amplify & $52.7 \pm 1.2$ & $52.4 \pm 1.6$ & $51.6 \pm 0.9$ \\
\hline Duso vs. Dusu & Scaled & $50.7 \pm 1.2$ & $52.4 \pm 1.6$ & $51.6 \pm 0.9$ \\
\hline & Amplify & $52.2 \pm 1.3$ & $53 \pm 1.2$ & $53 \pm 0.9$ \\
\hline & Scaled & $52.2 \pm 1.3$ & $53 \pm 1.2$ & $53 \pm 0.9$ \\
\hline & Amplify & $49.6 \pm 0.8$ & $50.2 \pm 1.0$ & $49.8 \pm 0.7$ \\
\hline D060 vs. DO 7 & Scaled & $49.6 \pm 0.8$ & $50.2 \pm 1.0$ & $49.8 \pm 0.7$ \\
\hline 0060 ys DO8 & Amplify & $49.1 \pm 0.8$ & $50.8 \pm 0.8$ & $50.9 \pm 1.2$ \\
\hline D060 vs. D08 & Scaled & $49.1 \pm 0.8$ & $50.8 \pm 0.8$ & $50.9 \pm 1.2$ \\
\hline & Amplify & $50.9 \pm 1.1$ & $52 \pm 0.9$ & $50.7 \pm 0.9$ \\
\hline & Scaled & $50.9 \pm 1.1$ & $52 \pm 0.9$ & $50.7 \pm 0.9$ \\
\hline & Amplify & $48.6 \pm 1.0$ & $49 \pm 1.0$ & $49.4 \pm 1.4$ \\
\hline Do/0 vs. Do & Scaled & $48.6 \pm 1.0$ & $49 \pm 1.0$ & $49.4 \pm 1.4$ \\
\hline & Amplify & $52.2 \pm 1.6$ & $49.5 \pm 0.9$ & $48.4 \pm 0.8$ \\
\hline & Scaled & $50.2 \pm 1.6$ & $49.5 \pm 0.9$ & $48.4 \pm 0.8$ \\
\hline D080 ys D & Amplify & $47.5 \pm 0.9$ & $48.9 \pm 1.1$ & $49.5 \pm 1.2$ \\
\hline Do80 vs. D & Scaled & $47.5 \pm 0.9$ & $48.9 \pm 1.1$ & $49.5 \pm 1.2$ \\
\hline
\end{tabular}

